\title{
Fine Needle Aspiration of Metastatic Metastatic Malignant in Parotid Gland: Case Report
}

\section{CASE REPORT}

\author{
Sibel Sensu ${ }^{1}$, Elife Kimiloglu' ${ }^{2}$ Ozgecan Gundogar ${ }^{2}$, Ceki Paltura ${ }^{3}$, Nusret Erdogan ${ }^{1}$ \\ ${ }^{1}$ Istinye University Medical Faculty Pathology Department, Istanbul - Turkey, ${ }^{2}$ University \\ of Health Sciences, GOP Taksim Research \& Education Hospital, Pathology Department, \\ Istanbul - Turkey, ${ }^{3}$ University of Health Sciences, GOP Taksim Research \& Education \\ Hospital, Ear, Nose and Throat Clinic, Istanbul - Turkey
}

\section{ABSTRACT}

Background : Malignant melanoma is the second most frequent tumor that metastasize to salivary glands. Fine needle aspiration (FNA) is a simple, rapid and widely accepted method for diagnosis of salivary gland lesions.

In this case report, forty-four years old male patient who admitted to hospital due to a painless mass in the parotid gland, is presented. A FNA was performed and following cytological, histological and immunohistochemical examination of the material, the final diagnosis was malignant melanoma. Deatiled patient history revealed a malignant melanoma of head and neck region which was diagnosed two years ago.

Although rare, metastasis of malignant melanoma to salivary glands has been reported in literature. Therefore, patients with primary tumor history should be carefully evaluated for metastasis. Besides, a malignant melanoma can be found in parotid gland in patients with an unknown primary locus. A regressed tumor should also be kept in mind.

Key Words: Fine needle aspiration, malignant melanoma, parotid gland

Received: 15 October 2019, Accepted: 28 November 2019.

Corresponding Author: Sibel Sensu, MD, Pathology Department, Istinye University Medical Faculty, Tel.: 05367844630,

E-mail: ssensu@yahoo.com

ISSN: 2090-0740, July 2020 Vol.21, No.2

\section{INTRODUCTION}

Primary malignant melanoma is a rather exceptional entity in parotid gland and malignant melanomas found in this location are mostly metastases of primary tumors of head and neck ${ }^{[1]}$ FNA is the most simple, rapid and valuable diagnostic tool for metastatic lesions in parotid gland. ${ }^{[2-4]}$

\section{CASE REPORT:}

Forty-four years old male patient admitted to hospital due to painless, rapid growing mass in parotid gland. Ultrasound examination was done and nearly $21 \times 13$ $\mathrm{mm}$, hypoechogenic solid mass with regular borders was diagnosed as pleomorphic adenoma. FNA was performed and cytological examination of alchoholfixed-Papanicolau-stained slides revealed diffusely dispersed round and spindle cells with large eosinophilic cytoplasm, prominent cytological atypia, nuclear grooves and pseudoinclusions (Figure 1). In hematoxylin-eosin stained cell blocks, pleomorphic cells with irregular nuclear contours, coarse chromatin and prominent nucleolus were forming discohesive clusters (Figure 2a). Immunohistochemical staining performed on cell block was very useful for diagnosis and positive cytoplasmic reaction was seen for Melan A, HBM-45 and S100 (Figure 2b) while no reaction was found for pancytokeratin, CD45 and SMA. Cytological, immunohistochemical findings and patient history were collectively evaluated and reported as "metastatic malignant melanoma". No other clinical and radiological evidence of metastatic disease was present.

Two years ago, a lesion excised form the scalp of the patient was histologically diagnosed as "nodular malignant melanoma" which had a Breslow thickness of $16 \mathrm{~mm}$ and Clark Level IV (reticular dermis invasion). Systemic evaluation had revealed no metastasis. Accordingly, tumor was stage T2a (AJCC 8 Ed.) and adjuvant therapy was not given. Informed consent was obtained from the patient prior to all diagnostic and therapeutical procedures. 


\section{DISCUSSION}

Malignant melanoma is an aggressive tumor with a high metastasis potential. Despite increase of surveillance and public awareness, its incidence is growing. ${ }^{[5]}$ National Cancer Institute's Surveillance, Epidemiology, and End Results (SEER) estimate that malignant melanoma will compose $5.5 \%$ of all new cancers and $1.2 \%$ of all deaths due to cancers in 2019. ${ }^{[6]}$ Malignant melanoma is the second most common tumor, after squamous cell carcinoma, that metastasize to salivary gland.$^{[7,8]}$ Nearly $25 \%$ of metastasis to salivary glands are derived from malignant melanomas. ${ }^{[9]}$

FNA is a very useful diagnosting tool for salivary gland lesions (86-98\% sensitivity and 97-98\% specificity) that supplies material for both cytologic and molecular assessments. Following FNA, cell block should be prepared since it is a good tool for immunohistochemical examination. ${ }^{[10,11]}$ However, in metastasis of malignant melanoma, clinical history is significant and in tumors with unknown primaries, successful diagnosis with FNA might be as low as $20 \%{ }^{[5]}$ Cellular slides with discohesive groups are characteristic for malignant melanoma and this discohesive pattern which differentiates it from epithelial tumors is due to loss of adhesion molecules. In malignant melanoma, round, oval, poligonal, spindle and some multinuclear cells are found and these are eosinophilic plasmocytoid-shaped pleomorphic cells with exantric nucleus and prominent nucleolus. ${ }^{[5,12]}$ Though intracytoplasmic inclusions might be seen in several neoplasia, multiple inclusions $<1$ micrometer are characteristic for malignant melanoma. Even if the most specific finding for malignant melanoma is presence of pigment, it is found in only half of the cases. Melanin pigment is best found in liquid based cytology, but is infrequent in conventional smears and tissue. ${ }^{[12]}$ Importantly, cell block should be prepared and patient history should be known for precise diagnosis. ${ }^{[11]}$ Al-Khafaji BM et al., investigated 154 FNA performed in parotid gland and found 5 malignant melanoma (4 correct positive diagnosis and 1 wrong positive diagnosis). ${ }^{[10]}$ In our case, patient history and immunohistochemical assessment (Melan A, HBM45 and S100 positivity in cell block) supported and facilitated our diagnosis.

Parotid gland malignant melanoma with unknown primary has a better prognosis and a longer diseasefree survival than cases with known primary. In a study of Prayson et al., ${ }^{[12]}$ malignant melanoma located in parotid gland was investigated; in 9 of the cases cutaneous or conjunctival malignant melanoma was found and survival was mean 22 months. ${ }^{[9]}$ A regressed primary malignant melanoma not readily recognizable should also be kept in mind. ${ }^{[9,13]}$ Our patient has a known primary tumor, is under follow up and diseasefree survival is 2 years.

As a conclusion, FNA is a simple and reliable diagnostic tool for malignant melanoma of salivary glands. Detailed evaluation of the case including both patient history and histomorphology will improve the accurate diagnosis.

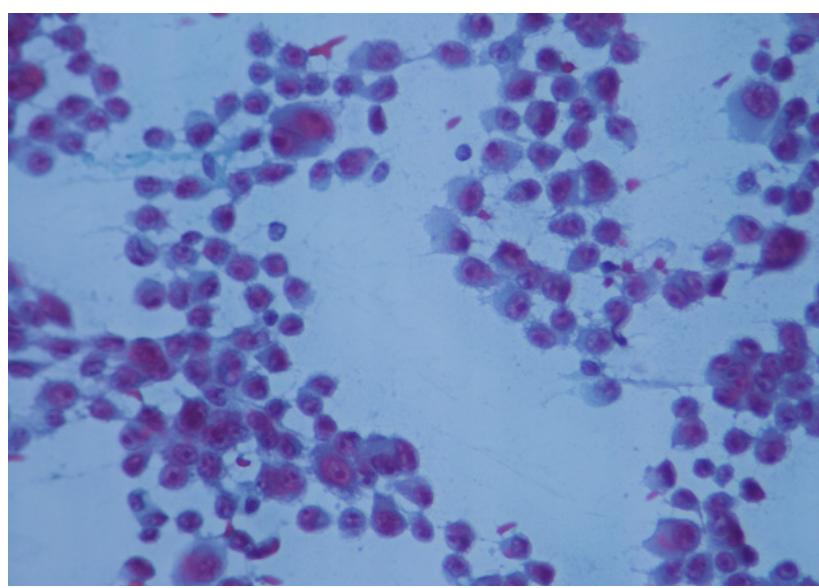

Fig. 1 : FNA: Malignant cells showing little cohesion, prominen nucleoli, pleomorphism and multinucleation. (PAP Stain X400).

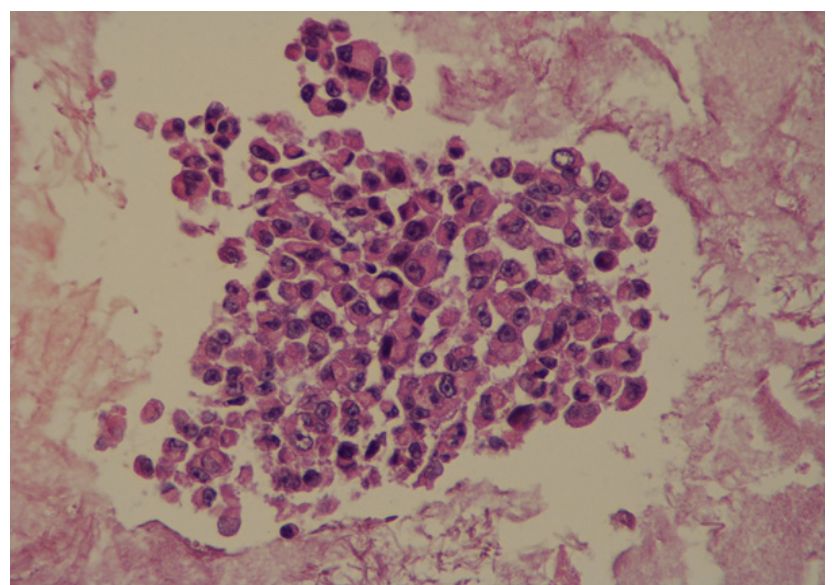

Fig. 2a : Cell block: A group of pleomorphic cells with irregular nuclear contours, coarse chromatin and prominent nucleolus. (HEx400).

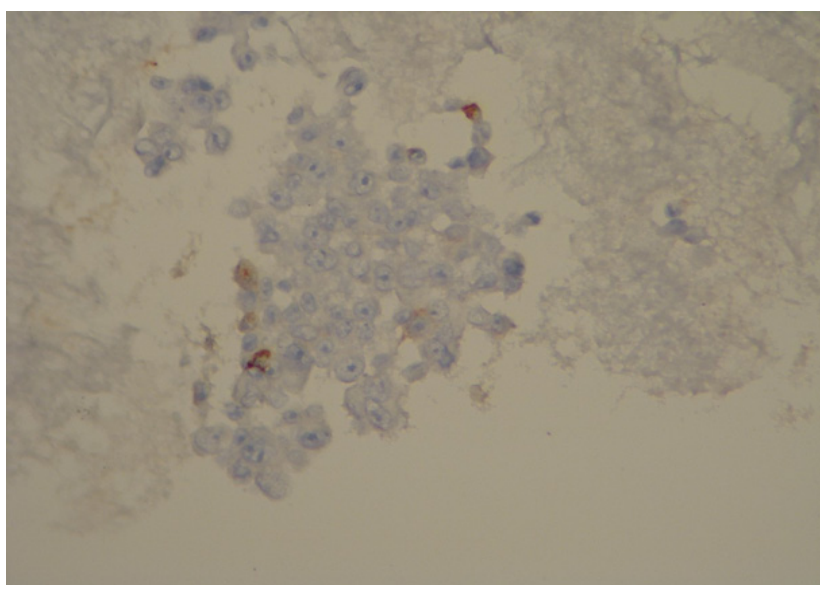

Fig. 2b : Cell block: A group of pleomorphic cells showing positive cytoplasmic reaction with HMB45.
(HMB45x400). 


\section{CONFLICT OF INTEREST}

There are no conflicts of interest.

\section{REFERENCES}

1. Andreadis D, Poulopoulos A, Nomikos A, et al. Diagnosis of metastatic malignant melanoma in the parotid gland. Oral Oncology Extra 2006;42(4):137139.

2. Zhang C, Cohen JM, Cangiarella JF, et al. FineNeedle Aspiration of Secondary Neoplasms Involving the Salivary Glands. A Report of 36 Cases. Am J Clin Pathol 2000;113(1):21-28.

3. Ersoz C, Uguz AH, Tuncer U, et al. Fine Needle Aspiration Cytology of the Salivary Glands: A Twelve Years' Experience. Agean Pathology Journal 2004; 1: $51-56$.

4. Wang H, Hoda RS, Faquin W, et al. FNA Biopsy of Secondary Nonlymphomatous Malignancies in Salivary Glands: A Multi-Institutional Study of 184 Cases. Cancer Cytopathol 2017;125(2):91-103.

5. Lindsey KG, Ingram C, Bergeron J, et al. Cytological diagnosis of metastatic malignant melanoma by fine-needle aspiration biopsy. Semin Diagn Pathol 2016;33(4):198-203.

6. https://seer.cancer.gov/statfacts/html/melan.html. Access date: June 2019
7. Franzen A, Buchali A, Lieder A. The Rising Incidence Of Parotid Metastases: Our Experience From Four Decades of Parotid Gland Surgery. Acta Otorhinolaryngologica Ital 2017;37(4):264-269.

8. Bron LP, Traynor SJ, McNeil EB, et al. Primary and Metastatic Cancer of the Parotid: Comparison of Clinical Behavior in 232 Cases. Laryngoscope 2003; 113(6):1070-1075.

9. Prayson RA, Sebek BA. Parotid Gland Malignant Melanomas. Arch Pathol Lab Med. 2000;124(12):1780-1784.

10. Al-Khafaji BM, Nestok BR, Katz RL. Fine Needle Aspiration of 154 Parotid Masses with Histologic Correlation: ten-year experience at the University of Texas M. D. Anderson Cancer Center. Cancer 1998;84(3):153-159.

11. Pusztaszeri MP, Garcia JJ, Faquin WC. Salivary Gland FNA: New Markers and New Opportunities for Improved Diagnosis. Cancer Cytopathol 2016;124(5):307-316.

12. Morrison C, Young DC, Wakely PE. Cytopathology of Malignant Melanoma in Conventional and LiquidBased Smears. Am J Clin Pathol 2002;118(3):435441.

13. Cengiz BP, Acar M, Aksay C, et al. Metastatic Melanoma of the Parotid Glands Bilaterally. West Indian Med J 2016; 65(2): 401-403. 